\title{
ECONOMIC POLICY FOR TOURISM DEVELOPMENT IN GEORGIA IN THE POST-CORONAVIRUS PERIOD
}

\section{GULNAZ ERKOMAISHVILI}

\author{
Doctor of Economics, Associate Professor, \\ Ivane Javakhishvili Tbilisi \\ State University, Georgia \\ gulnazi.erkomaishvili@tsu.ge
}

Abstract. The coronavirus pandemic has caused unprecedented shifts in the world economy. Especially the serious economic damage was caused to such countries, where tourism took great place in the economy. Georgia is one of such countries. 2019 in, 9,4 million tourists visited Georgia (\$3 ,3 billion revenue), 150 thousand people were employed in Tourism, and the share of tourism in GDP was $11,5 \%$ totally.

The paper presents the economic policy of tourism development in Georgia in the post-crisis period. To assist the tourism sector, the Georgian government has worked out an anti-crisis plan. The assistance program includes both anti-crisis measures and incentive tools. An indication of the allowances, that will be provided in the field of tourism employed in the business. In particular, in 2020, the tourism sector will be fully exempt from property taxes.

It is noted that in the post-crisis period, special attention should be paid to the promotion of domestic tourism, regional tourism, organized tourism from a number of countries, as well as medical tourism. During the pandemic, travel companies should be able to organize online tourism or virtual tours that will promote Georgiass tourist destinations.

The paper points out, that in spite of the fact that the Georgian government has always pursued a liberal policy for the development of the tourism sector, the tourism industry is still in its infancy. The development of this field in the country is hindered by many factors: in particular, the existence of conflict zones; High bank interest rates on loans, shortcomings in tax legislation; Despite the statess efforts, the country still lacks adequate infrastructure; The Quality of the service is low; there are unreasonably high prices which hinders the development of leisure tourism; there is no regulated statistical accounting, etc.

In 2020, we will probably not have a large number of foreign tourists in Georgia. During this period, quality infrastructure should be created, infrastructure and services in hotels and various tourist facilities should be improved, technologies and new management systems should be introduced, and tourism products should be created in new directions, including the development of national parks, reserves and protected areas.

In the post-crisis period, it is important to restore and develop agricultural processing enterprises, as this area is directly related to the development of tourism. A country dependent on imported products is unlikely to achieve proper tourism development. The state policy of tourism development is aimed at maintaining the liberalization sector and supporting it with investments.

KEYWORDS: TOURISM, TOURISM POLICY, POST-CORONAVIRUS PERIOD, ANTI-CRISIS PLAN FOR TOURISM DEVELOPMENT.

For citation: Erkomaishvili, G., (2020). Economic Policy for Tourism Development in Georgia in the Post-Coronavirus Period. Globalization and Business, 10. 257-260. (In Georgian) https://doi.org/10.35945/gb.2020.10.034 


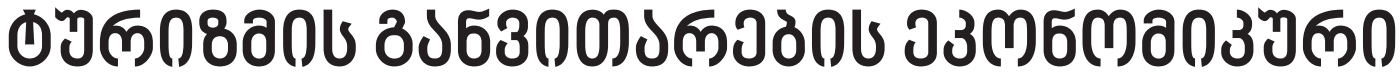

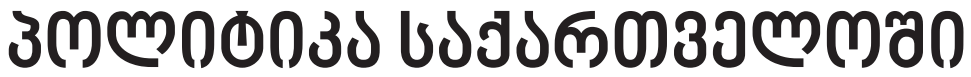

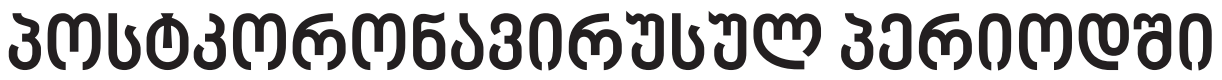

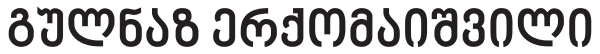

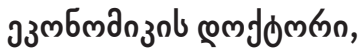

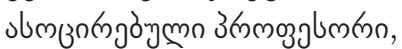

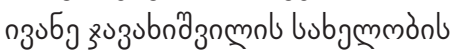

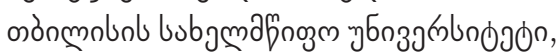 \\ bofurnom 3amm \\ gulnazi.erkomaishvili@tsu.ge
}

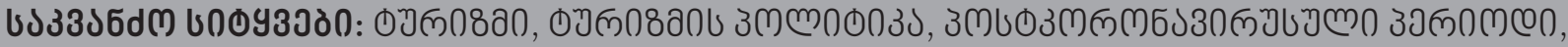

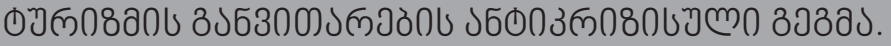

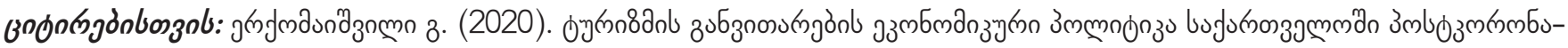

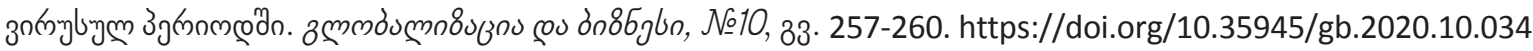

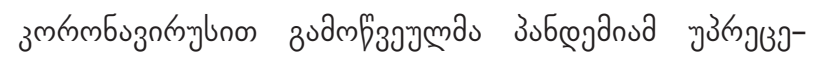

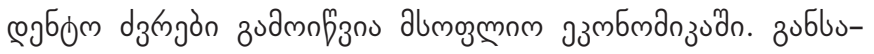

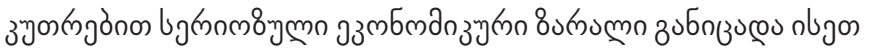

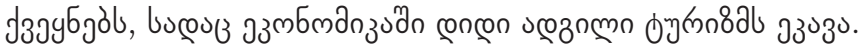

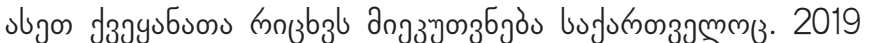

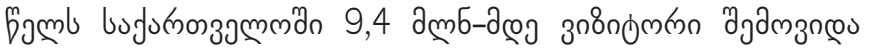

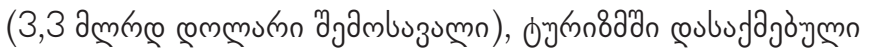

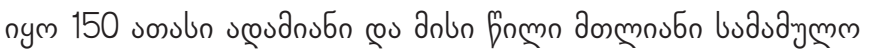

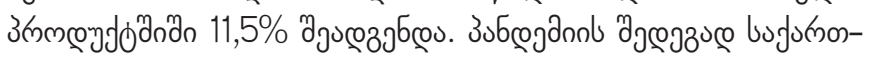

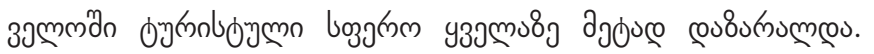

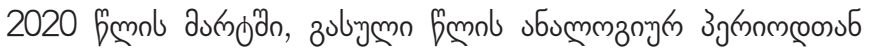

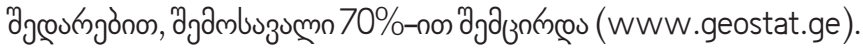

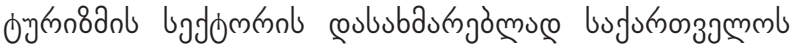

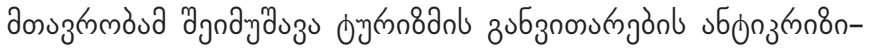

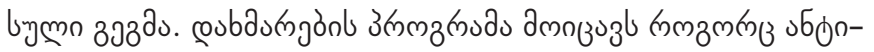

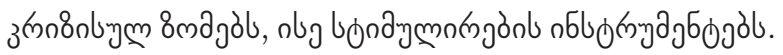

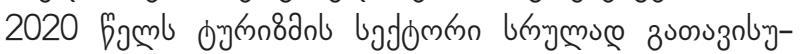

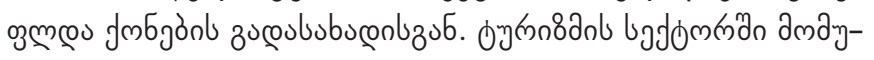

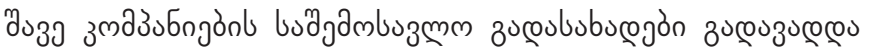

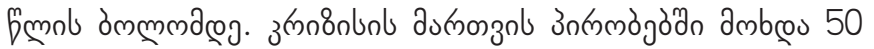

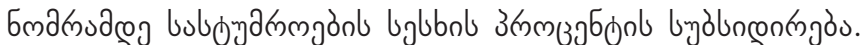

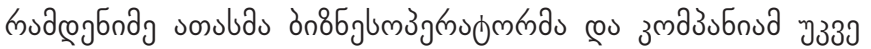

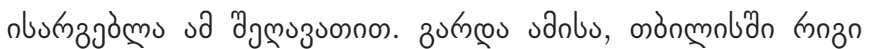

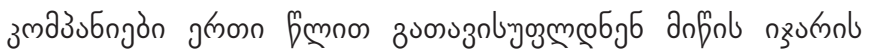

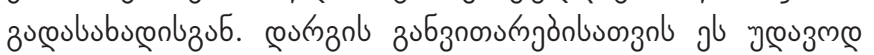

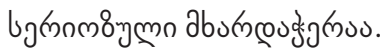

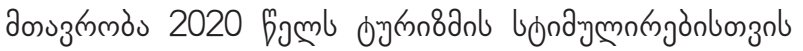

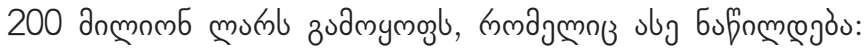

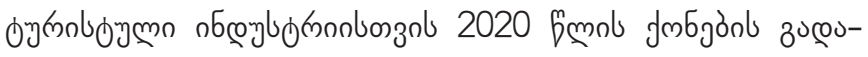

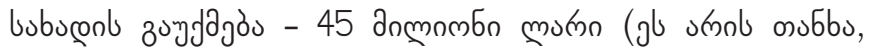

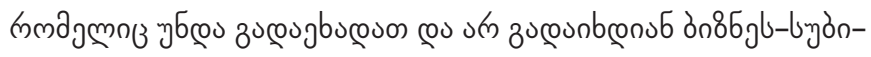

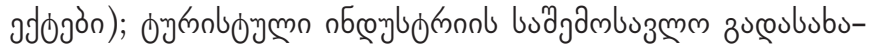

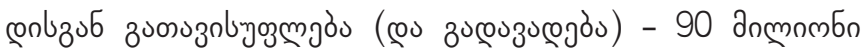

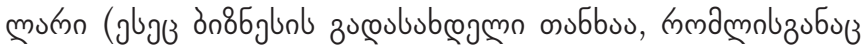

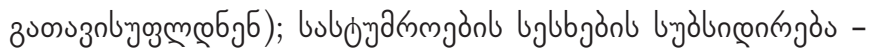

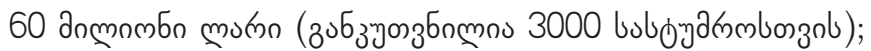

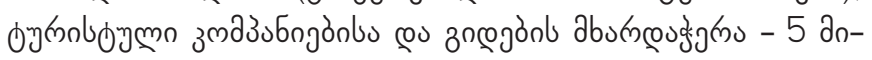

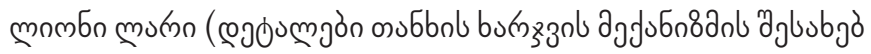

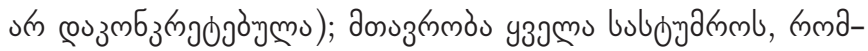

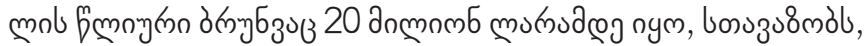

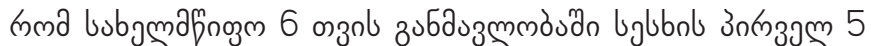

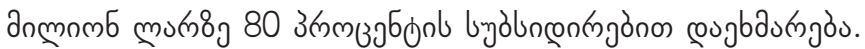

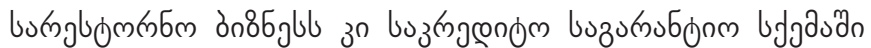

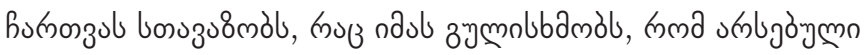

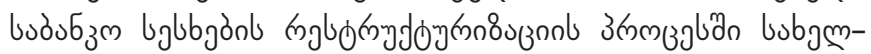

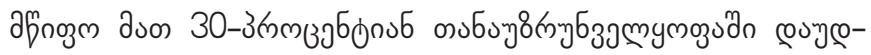

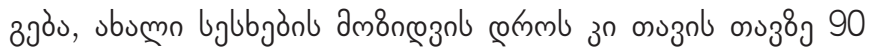

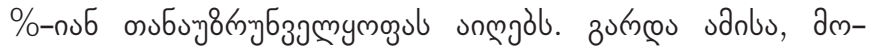

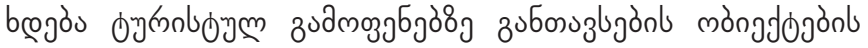

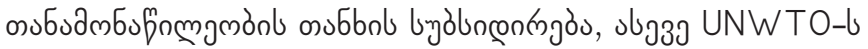

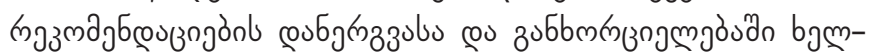

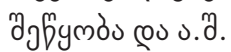

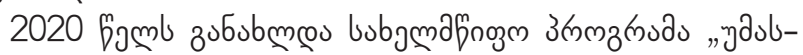

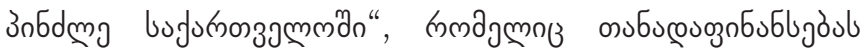

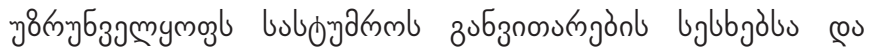

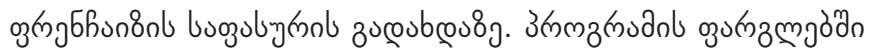




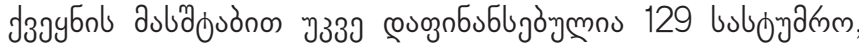

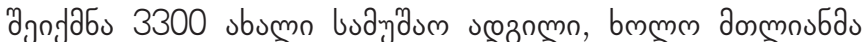

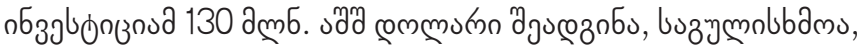

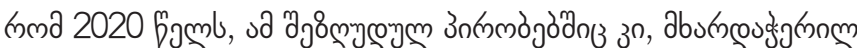

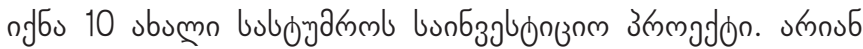

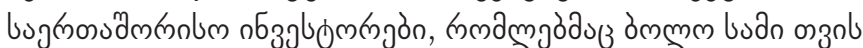

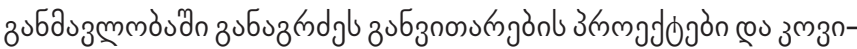

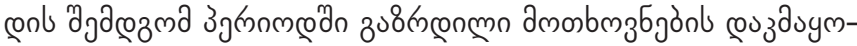

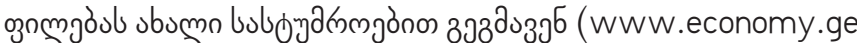
Anti-crisis plan for tourism).

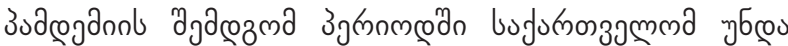
zudmng gб

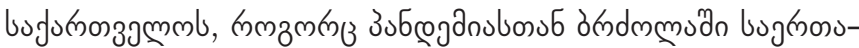

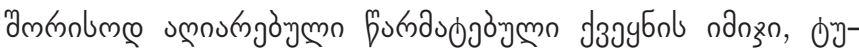

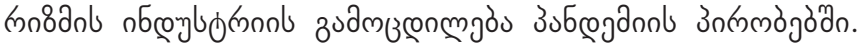

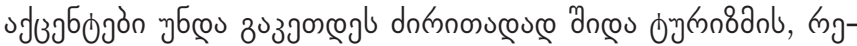

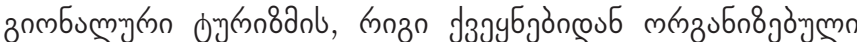

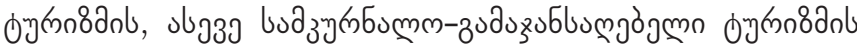

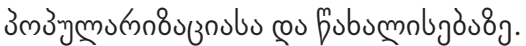

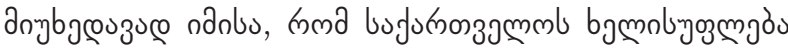

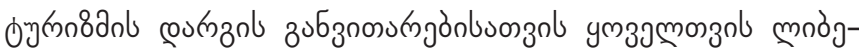

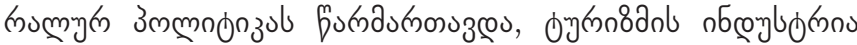

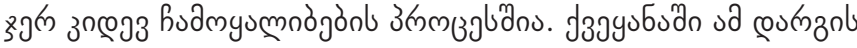

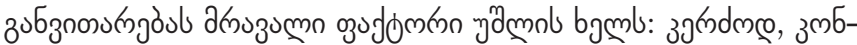

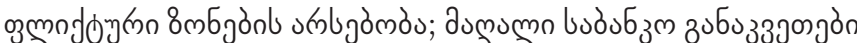

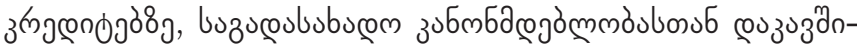

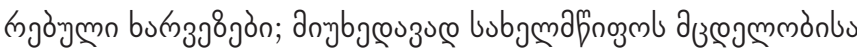

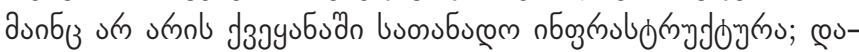

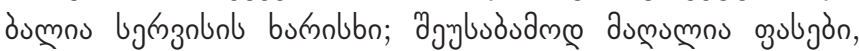

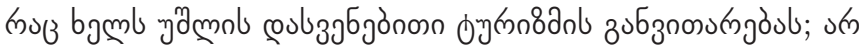

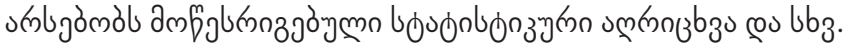

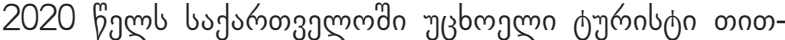

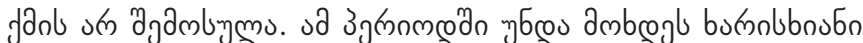

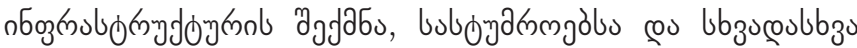

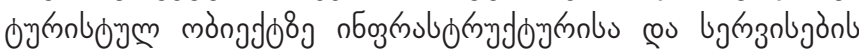

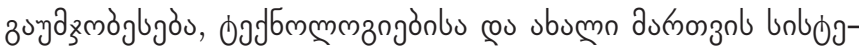

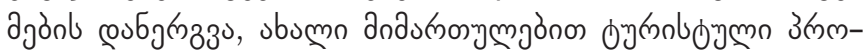

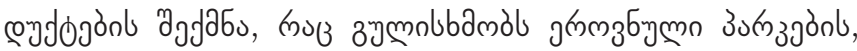

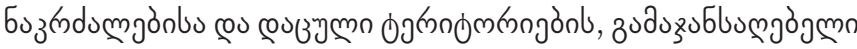

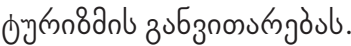

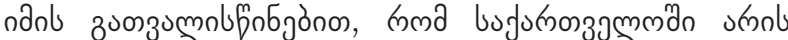

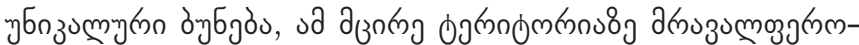

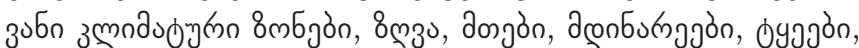

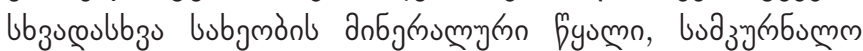

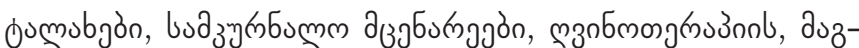

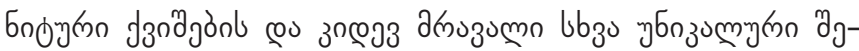

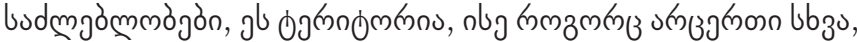

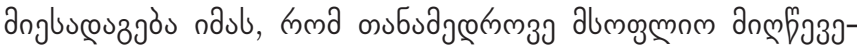

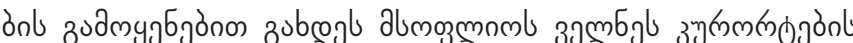
\3agubs. 3jm

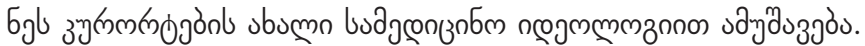

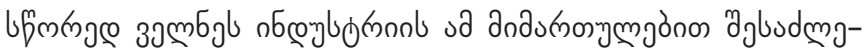

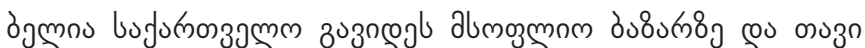

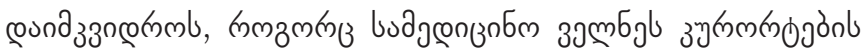

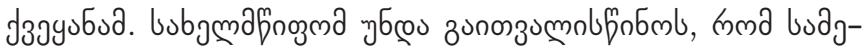

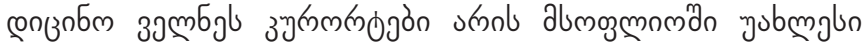

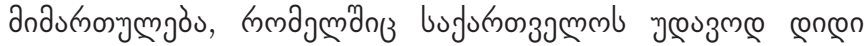

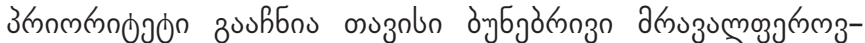

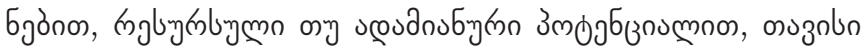

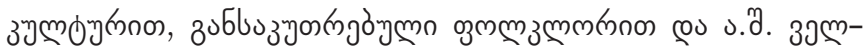

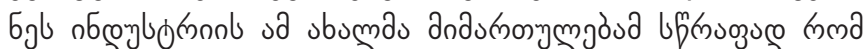

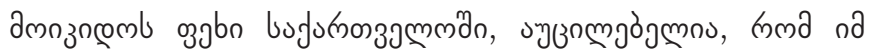

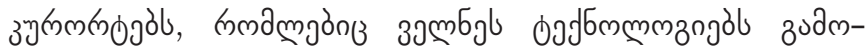

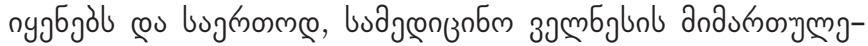

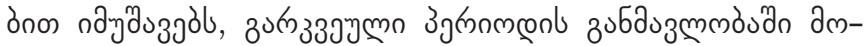

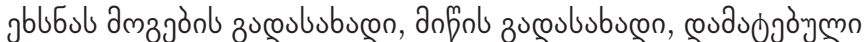

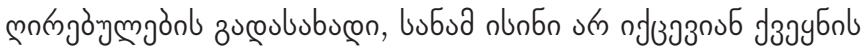

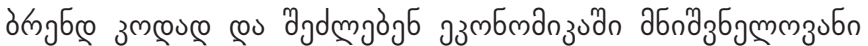
6зmomol ḋgoubsl (Erkomaishvili, 2019:108).

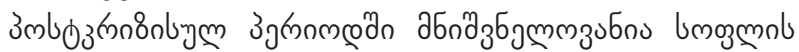

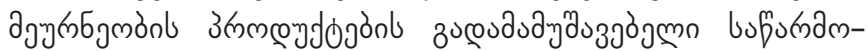
jön u uल@

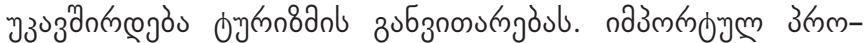

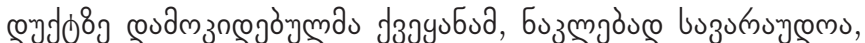

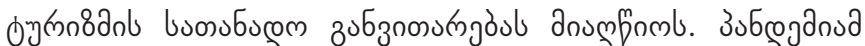

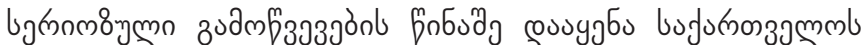

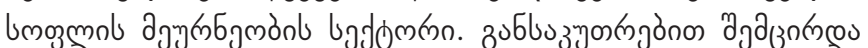

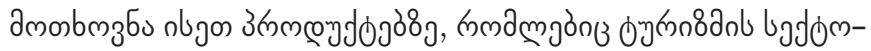

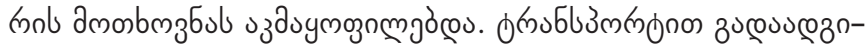

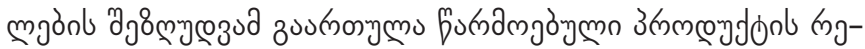

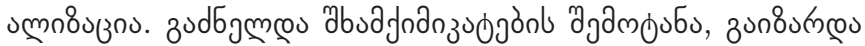
duon oुulon.

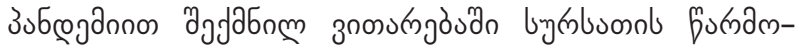

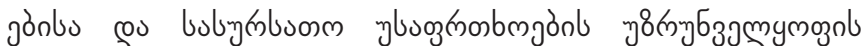

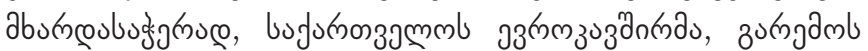

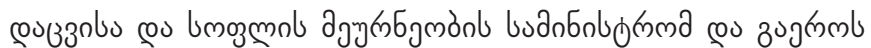

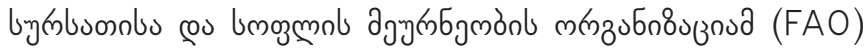

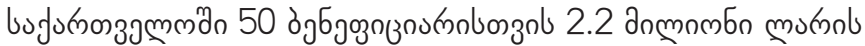

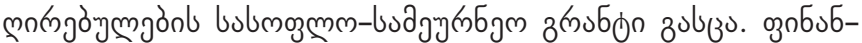

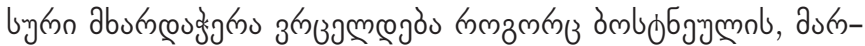

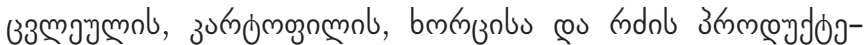

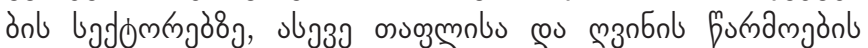

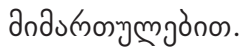

lmogmnl agy

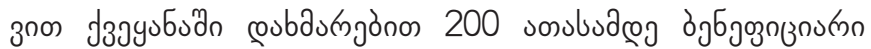

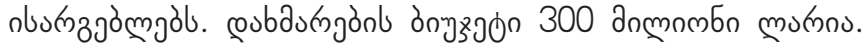

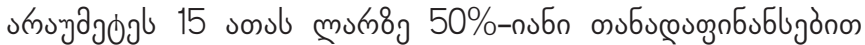

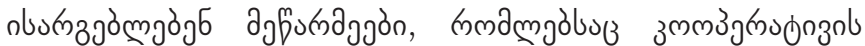

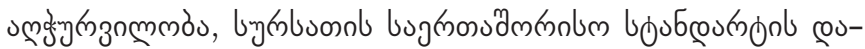




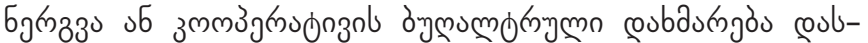

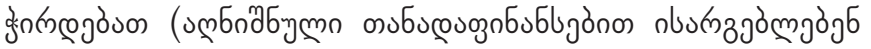

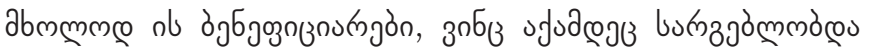

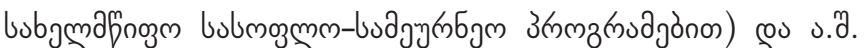
(www.economy.ge Anti-crisis plan for tourism).

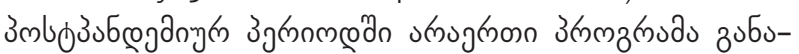

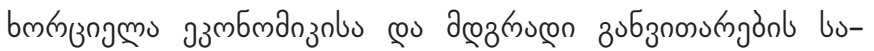

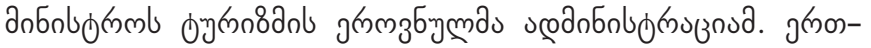

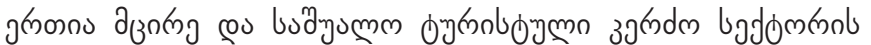

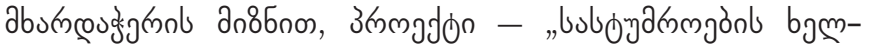

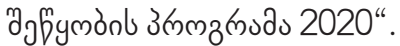

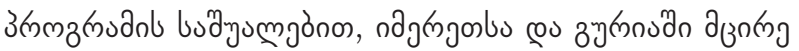

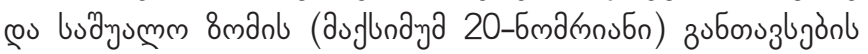

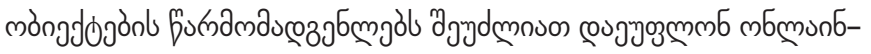

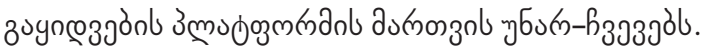

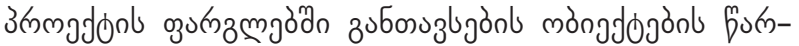
дмдам

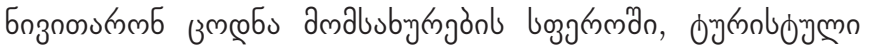

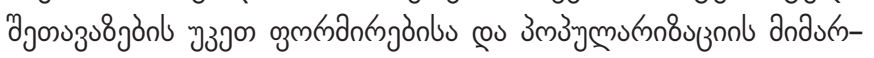

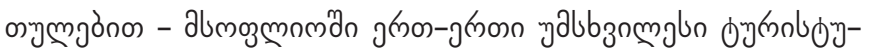

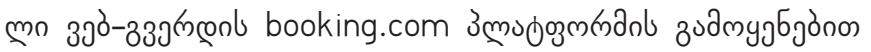
(www.gnta.ge).

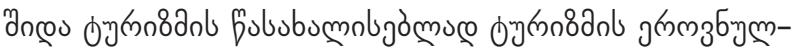

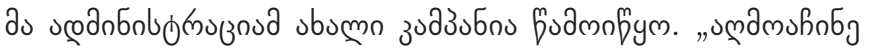

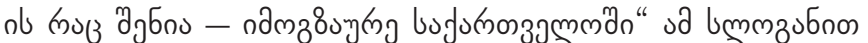

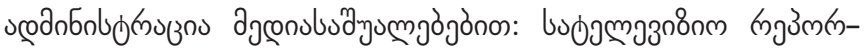

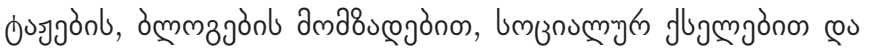

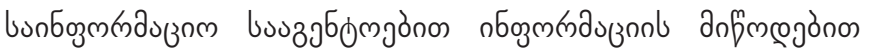

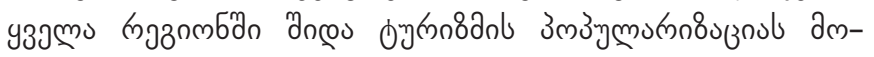

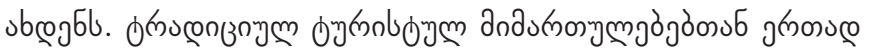

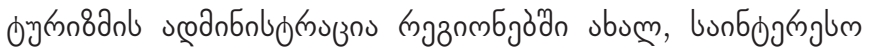

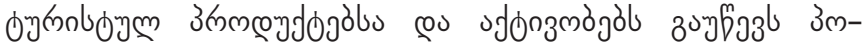

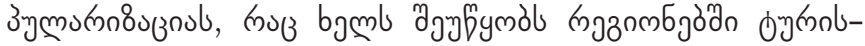

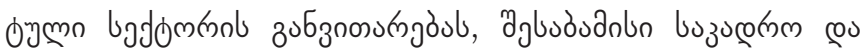

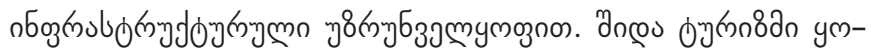

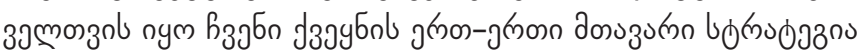

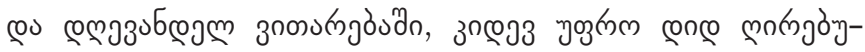

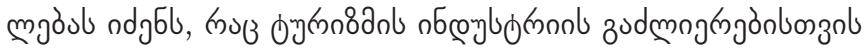
абпдзбаммलзибпо (www.gnta.ge).

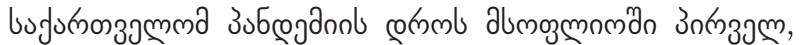

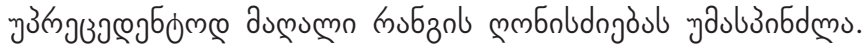

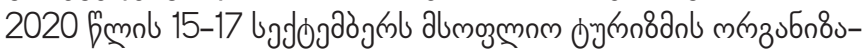

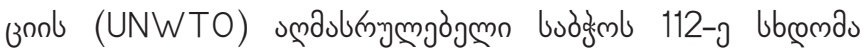

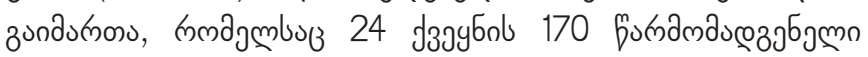

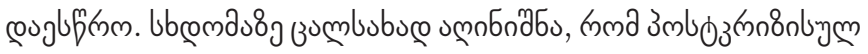

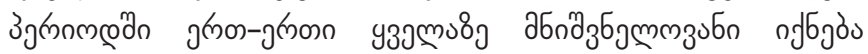

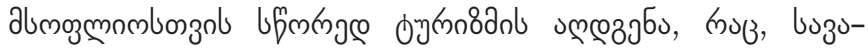

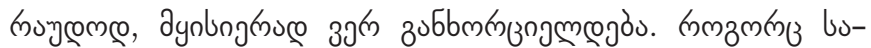

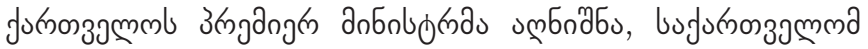

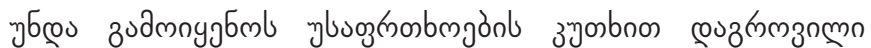

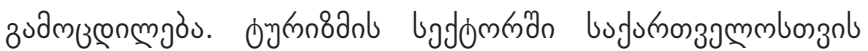

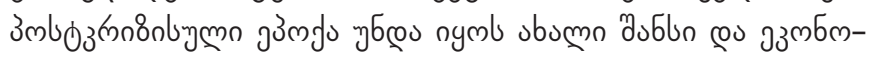

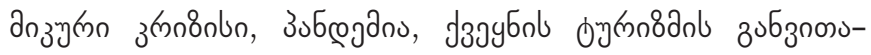

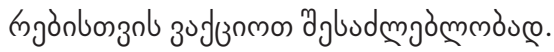

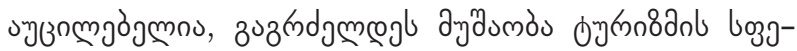

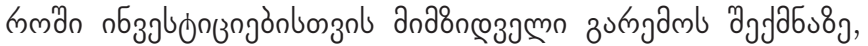

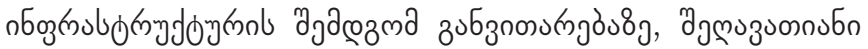

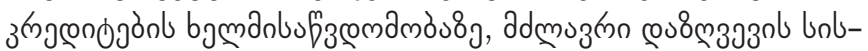

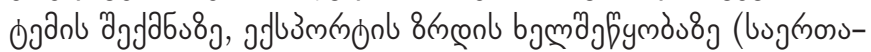
дmmolum du8

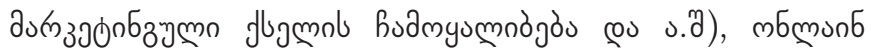

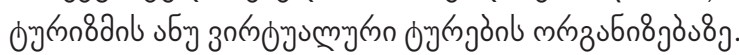

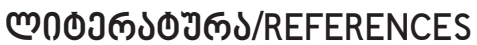

Erkomaishvili, G., (2019). Rational Economic Policy - Driving force from business to social entrepreneurship, Tbilisi.

Erkomaishvili, G., (2016). Economic Policy Priorities for Development of Georgia. Tbilisi.

Kharaishvili, E., Erkomaishvili G., \& Chavleishvili M., (2019). Trends of Agro-Food Production and Export Stimulating Economic Policy in Georgia, International Journal of Economics and Management Engineering, Vol:13, No:1, 2019.

Kharaishvili E., (2018). Diversification of Agribusiness and Rural Development Models in Georgia, IV International Scientific and Practical Conference - Strategic Imperatives of Modern Management.

www.geostat.ge

www.gnta.ge

www.economy.ge Anti-crisis plan for tourism. 